\title{
Spatiotemporal dynamics of natural phenomena
}

\author{
METMA4 workshop 2008
}

\author{
J. Mateu
}

Published online: 6 October 2009

(C) Springer-Verlag 2009

This special issue of SERRA is devoted to selected and revised papers presented at the fourth edition of the International Workshop on Spatio-Temporal Modelling (METMA4) that was held in Alghero, Italy, on 24-26 September, 2008.

In recent years, spatiotemporal modelling has become one of the most interesting and, at the same time, challenging research areas of natural sciences. The relevant literature is growing fast and along directions that range from theoretical works to methodological developments to real world applications. Spatiotemporal systems modelling involves the synthesis of a rich interdisciplinary body of knowledge for which it is necessary to establish a solid theoretical foundation and a science-based methodology with both researchers and practitioners in mind.

The purpose of this workshop was to promote the development and application of spatial, temporal, and mainly spatiotemporal statistical methods to different fields related to the environment. The workshop is organized every 2 years to bring together practitioners and researchers of different countries all over the world. The scientific program is characterized by having special invited sessions covering topics on the latest in theory, methods, and applications, as well as contributed papers and posters. There were finally 15 invited speakers, and delegates from more than 15 different countries, 85 participants in total. The scientific committee was of a great help to organize the program selecting papers for oral and/or poster presentations. The oral presentations were mainly given by invited speakers, although there were also some space in the

J. Mateu $(\bowtie)$

Department of Mathematics and IMAC, University Jaume I,

12071 Castellón, Spain

e-mail: mateu@mat.uji.es program for contributed papers. We also had a very interesting poster session.

We would like to express our gratitude to all the members of the local organizing and scientific committee. We also would like to thank all of the participants for their contributions to the spatiotemporal field in a wide sense: geostatistics, point processes, and lattice data. The workshop achieved the objectives of the organizing committee. There were no parallel sessions and this allowed nice discussions in the sessions, all of them well attended. The interaction among participants was high, and we hope that this will promote future collaborations.

The complete list of papers presented at the workshop and any particular information are posted at: http://www. uv.es/metma4.

In light of the above considerations, the articles of this special issue have been carefully selected to present a variety of conceptual frameworks, powerful methods and comprehensive techniques that address a number of interesting problems in environmental, health, social and medical sciences.

Mortality forecasts are nowadays widely used to create and modify retirement pension schemes, disability insurance systems and other social security programmes. During the 20th century, huge increases in life expectancy have followed medical and scientific breakthroughs. Forecasting mortality usually serves practical purposes, as improvements therein could have enormous social and financial implications. Dynamic tables analyze mortality data corresponding to a series of consecutive years. This approach allows to study the calendar effect's influence on mortality. The representation of the evolution of mortality via dynamic models is an important and timely issue, widely extended over actuaries, statisticians or demographers. Actually, a dynamic table can be considered as a two-way 
table on an equally spaced grid in either the vertical (age) or horizontal (year) direction. Under this framework, a dynamic table could be seen as a realization coming from a random field indexed by a point of $\mathbb{R}^{2}$ and whose coordinates are age and time (Martínez-Ruiz et al.). Thus, under this philosophy, the geostatistical approach may be an appealing alternative to the other models proposed by the literature, that are not taking into account the spatial dependence between the axis. Geostatistics is an important field in spatial statistics that can be used to model and further mimic spatiotemporal dynamics of many natural phenomena. In particular, this field constitutes a powerful means for generating rich families of spatiotemporal covariance and spectral density models.

Many environmental data are biased due to measurement's errors, and thus there is a need to assess sample bias. One possibility is modelling it using the skew-normal distribution, a generalization of the normal one. Advantages are twofold. In the first place, parameters of a skewnormal distribution have a meaningful interpretation from a decision maker's viewpoint. In the second place, the skewnormal distribution gives a very good fit to the data, especially in the tails, making it useful for estimation purposes. In particular, Bartoletti and Loperfido focus on the analysis of one of the most dangerous pollutants (especially in winter months), $P M_{10}$, that is $P M$ with an aerodynamic equivalent diameter of up $10 \mu \mathrm{m}$. The occurrence of a variety of adverse effects on health due to $P M_{10}$ has been reported consistently by hundreds of epidemiological studies of different designs (such as metaanalyses, multi-city and single-city studies). Although the biological mechanisms through which PM10 affects health are only partially understood, toxicological evidence strongly corroborates the associations observed in epidemiological studies. Several scientific studies agree about the harmful effects of $P M_{10}$ on human health, vegetation and manufactured materials, in proportion to high concentrations. Low concentrations of $P M_{10}$ are dangerous, too: most epidemiological studies on large populations have been unable to identify a threshold concentration below which $P M_{10}$ has no effect on mortality and morbidity.

In environmental applications it is often important to analyze the seasonality of a time series as well as the remaining seasonally adjusted time series. In fact, some researchers claim that seasonality is some form of data contamination that can be removed from the data in order to facilitate the analysis of, for example, trends and business cycles. In a more general sense, the last decades have witnessed a growing interest in extracting accurately the periodic components from a time series. Mouri'no and Bar'ao focus on a generic time series whose behaviour is ruled by a periodic structure superimposed on a serially correlated stochastic process. In order to analyze the effects of selecting different formulations to accommodate the autocorrelation in the residuals, they consider two seemingly different ways to deal with this problem: the Linear Regression Model with the sequence of error terms following an Autoregressive Stationary Process and the Partial Adjustment Model. Comparing the statistical properties of these modelling schemes, they are able to discuss the strengths and weaknesses of fitting the former model, or, on the other hand, to rewrite the design matrix by introducing lagged dependent variables.

A wide variety of dynamical biological, geophysical and environmental systems have been described through pseudodifferential evolution models. Ruiz-Medina and Fernández-Pascual consider the case where functional sample information is available from such systems. Specifically, the observation model is defined in terms of a sequence of spatial realizations of the process of interest, solution to a spatiotemporal pseudodifferential equation, affected by additive strong Hilbertian white noise. Conditions for a stable computation of the solution to the associated functional filtering problem are established in terms of the covariance operator spectra of the process of interest and of the Hilbertian observation noise. In practice, such conditions are referred to the empirical spectra associated with the covariance operator estimators.

In aquatic ecosystems a crucial control on carbon processing is exerted by the animals inhabiting the sediments, which extensively rework and oxygenate sediments while e.g. feeding and moving, a global process referred to as bioturbation. In particular, macrofauna, through their feeding, burrowing and ventilation activities, have an important influence on microbial activity and sediment metabolism in marine sediments. Subduction of oxygen into and removal of metabolites from otherwise anoxic sediments, as well as relocation of organic particles by the infauna, create a heterogeneity, which can result in several-fold enhanced rates of organic matter decomposition and solute fluxes. Overall, bioturbation plays a key role in the ecosystem functioning. One way to investigate this role is to study the related oxygen heterogeneity. For this purpose the use of planar optodes makes possible high resolution measurement of twodimensional vertical distribution and spatial heterogeneity of oxygen in the sedimentary column.

Dabo-Niang et al. provide a useful tool to compare the effects of bioturbation on the distribution of oxygen within sediments. They propose heterogeneity measurements based on functional spatial mode. To obtain the mode, one usually needs to estimate the spatial probability density. The approach considered consists in considering each observation as a curve that represents the history of the oxygen concentration at a fixed pixel. 
Gutiérrez et al. consider the two parameter Gompertz diffusion process that can be used to model growth phenomena defined on a subset of a plane. This diffusion version of the Gompertz stochastic model constitutes a model of great interest to investigators of several fields, including demography, biology, economics and environmental sciences. These authors obtain some analytical properties of the transition density of the spatial Gompertz diffusion by an appropriate transformation of a Gaussian diffusion field. They estimate the parameters of the spatial diffusion when the observations are taken on a regular grid. In addition they try to derive the estimate of the parameters on an irregular grid, and for some parameters they are able to obtain explicit expressions.

We finally would like to thank the authors for their outstanding research contributions and, in particular, Prof. George Christakos (Stochastic Environmental Research and Risk Assessment, Editor-in-Chief) for his encouragement and support in preparing this special issue. 